\title{
VALIDAÇÃO APARENTE E DE CONTEÚDO DE UMA CARTILHA DE AUTOCUIDADO PARA PREVENÇÃO DE LESÃO POR PRESSÃO
}

\section{APPLICABLE AND CONTENT VALIDATION OF A SELF-CARE CARE BOOKLET FOR PRESSURE INJURY PREVENTION}

\section{VALIDACIÓN APARENTE Y DE CONTENIDO DE UNA CARTILLADE AUTOCUIDADO PARA LA PREVENCIÓN DE LESIONES POR PRESIÓN}

Isabela Rodrigues Ferreiraํㅜ, Laysla Luiza Santos², Juliano Teixeira Moraes ${ }^{3}$, Daniel Nogueira Cortez ${ }^{4}$

\section{RESUMO}

Objetivo: Descrever o processo de construção e validação de uma cartilha de autocuidado para prevenção de Lesão por Pressão (LP). Método: Trata-se de uma pesquisa metodológica, com abordagem quantitativa, que construiu uma cartilha de autocuidado para prevenção de pacientes com risco para desenvolver LP. A pesquisa foi desenvolvida em 4 etapas: levantamento bibliográfico; construção do material educativo (ilustração, layout, design e textos), cálculo do índice de Facilidade de Leitura de Flesch e validação do material por experts da área. Resultados: A cartilha foi validada quanto ao conteúdo e aparência por 11 experts, com o Índice de Validade de Conteúdo (IVC) de 0,93\%. Os textos da cartilha foram avaliados como de leitura, razoavelmente fácil, com Índice de Facilidade de Leitura de Flesch (IFLF) de 71\%. Conclusão: A cartilha foi validada quanto ao conteúdo e aparência, podendo ser usada como material educativo aos pacientes que apresentam risco de desenvolver LP.

Descritores: Lesão por Pressão; Autocuidado; Folheto.

\section{ABSTRACT}

Objective: To describe the process of creation and validation of a self-care booklet for the prevention of Pressure Injury (PI). Method: This is a methodological research with a quantitative approach, which built a self-care primer to prevent patients at risk for developing PI. The research was developed in four stages: bibliographic survey; construction of educational material (illustration, layout, design and texts), calculation of the Flesch Readability Index and validation of the material by experts in the field. Results: The booklet was validated for content and appearance by 11 experts, with a Content Validity Index (CVI) of $0.93 \%$. The booklet texts were rated as reasonably readable, with a Flesch Readability Index (IFLF) of $71 \%$. Conclusion: The booklet has been validated for content and appearance and can be used as educational material for patients at risk of developing PI. Keywords: Pressure Ulcer; Self Care; Booklets.

\section{RESUMEN}

Objetivo: Describir el proceso de construcción y validación de una cartilla de autocuidado para la prevención de lesiones por presión (LP). Método: Esta es una investigación metodológica, con un enfoque cuantitativo, que construyó un manual de autocuidado para prevenir a los pacientes en riesgo de desarrollar LP. La investigación se desarrolló en 4 etapas: encuesta bibliográfica; construcción de material educativo (ilustración, diseño, diseño y textos), cálculo del índice de legibilidad de Flesch y validación del material por expertos en la materia. Resultados: El folleto fue validado por el contenido y la apariencia por 11 expertos, con un índice de validez de contenido (CVI) del 0,93\%. Los textos del folleto fueron calificados como razonablemente legibles, con un índice de legibilidad Flesch (IFLF) del 71\%. Conclusión: El folleto ha sido validado por su contenido y apariencia y puede usarse como material educativo para pacientes con riesgo de desarrollar LP.

Descriptores: Úlcera por Presión; Autocuidado; Folletos.

${ }^{1}$ Enfermeira pela Universidade Federal de São João Del Rei - UFSJ. ${ }^{2}$ Enfermeira pela UFSJ. ${ }^{3}$ Doutor em Ciências Aplicadas à Saúde do Adulto. Professor adjunto do curso de Enfermagem da UFSJ. " Doutor em Enfermagem e Saúde. Professor adjunto do curso de Enfermagem da UFSJ.

\section{Como citar este artigo:}

Ferreira IR, Santos LL, Moraes JT, et al. Validação aparente e de conteúdo uma cartilha de autocuidado para prevenção de lesão por pressão. Revista de Enfermagem do Centro Oeste Mineiro. 2020;10:e3648. [Access ]; Available in: DOI: 


\section{INTRODUÇÃO}

O perfil da população mundial tem mudado nos últimos anos. O aumento da expectativa de vida e os avanços da área da saúde modificaram o padrão de doenças, representado pelo aumento das taxas de incidência e prevalência de doenças crônicas. Essa mudança no perfil epidemiológico elevou o número de indivíduos idosos na população, as taxas de desnutrição e as taxas de internações hospitalares. Essas características apresentam-se como fatores de risco para comorbidades com maior prevalência entre os idosos, com destaque para as Lesões por Pressão $(\mathrm{LP})^{(1)}$.

Em razão da alta incidência e prevalência dessas lesões e melhor abrangência do termo, em 2016 a National Pressure Ulcer Advisory Panel (NPUAP) uma organização norte-americana dedicada à prevenção e tratamento de lesões por pressão, realizou a adequação do termo úlcera por Pressão para Lesão por Pressão. De acordo com essa entidade, geralmente, a LP acomete a pele e/ou tecidos moles subjacentes sobre uma proeminência óssea ou está relacionada ao uso de dispositivo médico ou a outro artefato e é proveniente da pressão severa e/ou por tempo extenso em associação ao cisalhamento e pode se apresentar em pele íntegra ou como úlcera aberta que pode ser dolorosa ${ }^{(2)}$.

Considerada como um evento adverso, a LP é uma das cinco causas mais comuns de danos ao paciente, conforme os parâmetros da Política Nacional de Segurança do Paciente, lançada, em 2013, no Brasil ${ }^{(3-5)}$. A prevenção e o tratamento da LP são multidisciplinares e envolvem ações terapêuticas e educativas, pois visam a corrigir os fatores de risco para o desenvolvimento das lesões, assim como realizar a sua cicatrização. Destaca-se que essas ações passam por solucionar os principais agentes causadores: a pressão intensa e o cisalhamento ${ }^{(6)}$.

Em relação às ações educativas, o sucesso na prevenção e tratamento da LP envolve também a participação do paciente, dos seus familiares/cuidadores e dos profissionais de saúde. ${ }^{(7)}$ Nesse contexto, existem diversas tecnologias e formatos de instrumentos educativos, como folhetos, manuais, folders, livretos, álbum seriado e cartilhas que podem se apresentar impressos ou de forma virtual para o uso em aplicativos, mas que ainda não foram produzidos e validados cientificamente. No entanto, a literatura é escassa para materiais dessa natureza voltados para LP. Espera-se que esses materiais possam despertar o interesse do indivíduo, fortalecer a fixação do que foi orientado, verbalmente, além de permitirem o acesso fácil para sanar dúvidas e auxiliar as ações cotidianas $^{(8)}$. Sendo assim, o objetivo do estudo foi descrever a construção e validação de uma cartilha de autocuidado para prevenção de LP.

\section{METODOLOGIA}

Trata-se de uma pesquisa metodológica, com abordagem quantitativa, que construiu uma cartilha de autocuidado para a prevenção de LP. Este estudo foi desenvolvido em 4 etapas: levantamento bibliográfico; construção do material educativo (ilustrações, layout, design e textos), cálculo do índice de Facilidade de Leitura de Flesch (IFLF) e validação do material por experts da área. Na primeira etapa, foi realizado levantamento bibliográfico sobre cartilhas e textos que abordassem o tema Lesão por Pressão. Este foi feito nas bases de dados Biblioteca Virtual em Saúde e PubMed, utilizando os descritores presentes no Descritores em Ciência da Saúde/Medical SubjectHeadings (DeCS/MeSH): "Lesão por Pressão" ("PressureUlcer", "Autocuidado" ("Care Self"), "Folheto" ("Pamphlet"). Testaram-se outros descritores nas bases descritas, sem a identificação de estudos com Lesão Por Pressão. Utilizou-se o descritor controlado "Lesão por Pressão" ("PressureUlcer") associado, por meio do operador booleano AND aos descritores supracitados e OR aos seus respectivos MeSH. Para análise foram incluídos os trabalhos dos últimos 10 anos, publicados de 2009 a 2019 e não foram encontrados estudos que associassem diretamente ao tema proposto.

Na segunda etapa, as ilustrações da cartilha foram elaboradas por um desenhista, a partir das orientações e requerimentos dos pesquisadores. Concomitantemente, com a criação das ilustrações, os pesquisadores elaboraram os textos da cartilha sobre LP e modo de prevenção.

$\mathrm{Na}$ terceira etapa, os textos do material educativo foram avaliados, a partir do Índice de Facilidade de Leitura de Flesch, tal índice de legibilidade é um modelo matemático que avalia a estrutura de um texto quanto às suas frases, parágrafos e quantidade de sílabas das palavras (tamanho) e é uma das fórmulas de legibilidade mais utilizadas, atualmente, considerada adequada para todos os tipos de textos ${ }^{(9)}$.O IFLF avalia o grau de legibilidade dos textos em uma escala porcentual de zero a cem e o seu resultado 
classifica o texto, de acordo com a dificuldade de leitura. Neste estudo, os textos foram selecionados e avaliados pelo programa de análise de textos do Microsoft Office Word 2013. Um texto considerado padrão pelo IFLF aquele com percentuais de 60 a $70 \%$, de acordo com o Escore de Flesch ${ }^{(10-11)}$.

A quarta etapa seguiu os princípios da técnica Delphi, para validação por experts no assunto quanto ao conteúdo e aparência da cartilha, no período de março a abril de 2019. No que se refere à validade do conteúdo, utilizou-se o Índice de Validade de Conteúdo (IVC), que afere a proporção de juízes que concordam sobre certos aspectos do instrumento e de seus itens, que analisa, individualmente, cada item e, posteriormente, o material educativo como um todo. Para isso, empregamos uma escala do tipo Likert com pontuação de um a quatro, que garante uma consistência interna satisfatória ${ }^{(12)}$. Segundo Pasquali ${ }^{(13)}$, existem doze critérios para a avaliação de instrumentos, que baseados em suas características, adotaram-se os seguintes: clareza, objetividade e relevância, tanto dos textos quanto das imagens. Para a análise dos textos, os experts foram orientados a considerarem linguagem como adequada para o público alvo. As respostas seguiram o seguinte critério para avaliação: $1=$ não representativo, $2=$ item necessita de grande revisão para ser representativo, $3=$ item necessita de pouca revisão para ser representativo e $4=$ representativo. Os itens pontuados como 1 e 2 foram revisados ou eliminados. Dessa forma, o IVC foi calculado pela proporção de itens que receberem uma pontuação 3 e 4 pelos juízes. A fórmula para avaliar cada item, individualmente, é a seguinte: IVC= número de respostas 3 e 4, dividido pelo número total de respostas. Para determinar o índice válido de concordância, alguns autores sugerem uma concordância mínima de 0,80 . No entanto, em construção e validação de novos instrumentos o índice recomendado é de 0,90 ou mais. Foi considerado como parâmetro de validade $\mathrm{o}$ índice de concordância de $80 \%{ }^{(14)}$.

A concordância entre os juízes foi calculada, por meio da fórmula: \% concordância = número de participantes que concordam, dividido pelo número total de participantes, multiplicado por 100. Foram considerados experts profissionais que apresentem, pelo menos dois dos seguintes critérios: desenvolvimento de ações de prevenção e/ou promoção da saúde nos últimos 10 anos, voltadas para pessoas com LP; ter trabalhos científicos publicados sobre LP e/ou sobre construção e validação de materiais educativos; ser especialista em estomaterapia e/ou membro da Sociedade Brasileira de Estomaterapia; ser mestre ou doutor com produção científica na área de LP ou produção de tecnologia educativa. Para identificação dos experts, o Currículo Lattes foi consultado para verificar a adequação do expert aos critérios estabelecidos no estudo. As alterações sugeridas pelos profissionais experts foram consideradas na versão final do material educativo. Os profissionais experts foram convidados a participar do estudo, por meio do contato por e-mail e utilizou-se uma plataforma online E-survey para coletar as avaliações dos juízes experts. Para participação na pesquisa todos os participantes assinaram o Termo de Consentimento Livre Esclarecido (TCLE).

O número de experts que participaram da pesquisa foi determinado por conveniência, sendo que esse número não poderia ser inferior a seis profissionais ${ }^{(15)}$.

A presente pesquisa foi aprovada pelo Comitê de ética em Pesquisa da Universidade Federal de São João del-Rei (UFSJ) Campus Centro Oeste Dona Lindu sob o parecer no 158.505 e CAAE 07330012.8.0000.5545.

\section{RESULTADOS E DISCUSSÃO}

$\mathrm{Na}$ busca de estudos que tratassem sobre construção e validação de instrumentos educativos, foram encontrados 18 artigos que pudessem abordar sobre o tema. Após a leitura completa dos mesmos, quatro relacionavam-se à construção e validação de cartilhas educativas, mas nenhum se referia à $L P$.

A cartilha intitulada "Cartilha de Autocuidado para Prevenção de Lesão por Pressão", foi composta por capa, 5 páginas e 19 imagens, sendo que cada página contou com uma média de 4 imagens. Na construção de materiais educativos reforça-se a necessidade de que os mesmos sejam interativos, atraentes e possuam linguagem adequada ao público alvo, proporcionando a troca de informações relevantes e contextualizadas ${ }^{(16)}$. As imagens da cartilha são bem detalhadas para que indivíduos que não saibam ler consigam interpretar e colocar em prática as sugestões de cuidados para prevenção de Lesão por Pressão (Figura 1). 
Figura 1 - Representação ilustrativa da cartilha educativa apresentada aos juízes. Divinópolis, 2019.
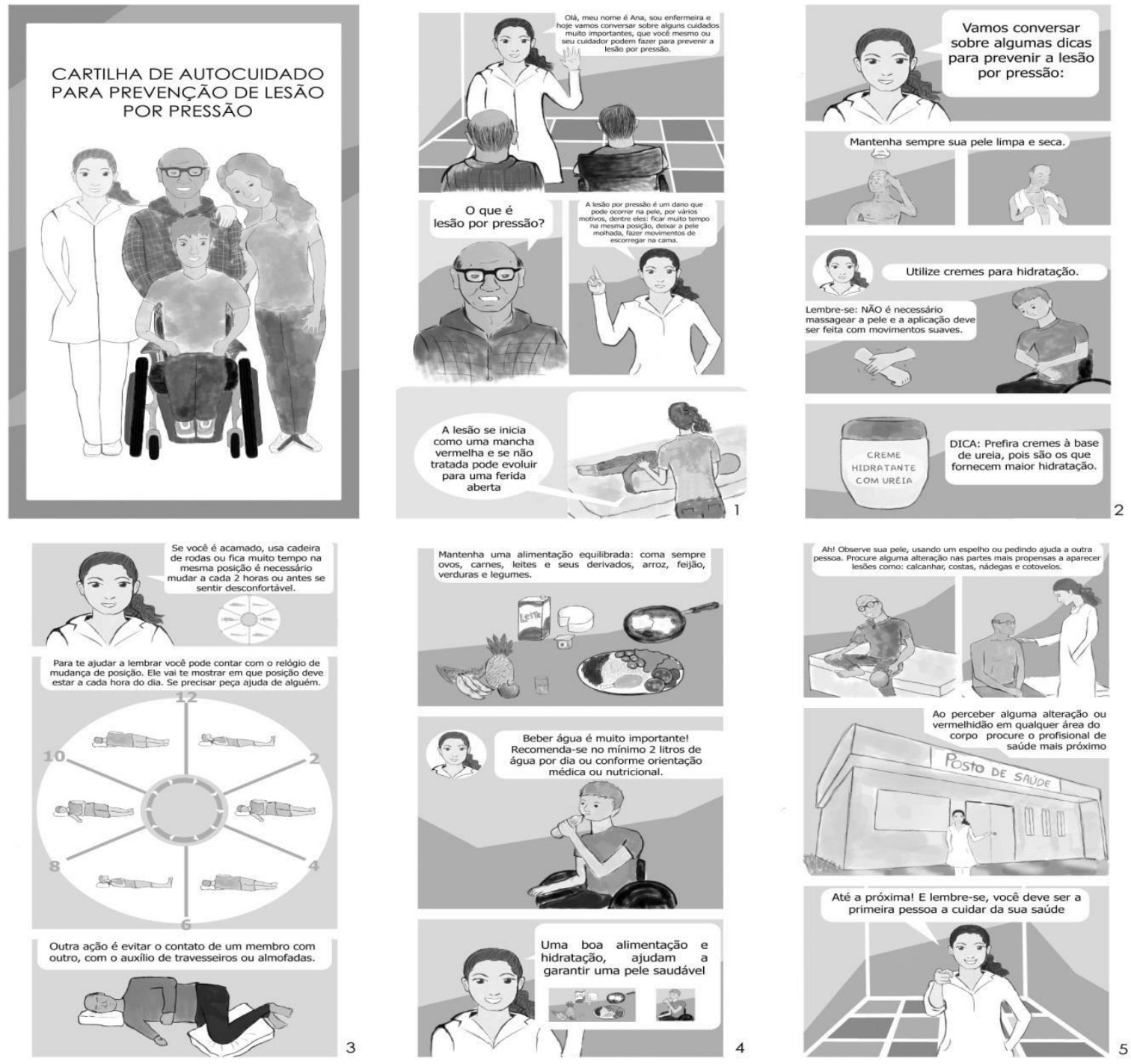

Fonte: Elaborada pelos autores.

A validação aparente da cartilha foi considerada adequada pelos experts, que sugeriram pequenas alterações de concordância verbal, adição ou mudança de imagens e troca de terminologia nas cinco páginas do instrumento. Isso significa que, numa análise semântica, todos os itens são compreensíveis para todos os membros da população a que se destina ${ }^{(17)}$.

Os textos do material educativo foram elaborados pelos pesquisadores e avaliados a partir do IFLF.

O valor do IFLF obtido, após cálculo, foi de $71 \%$, o que avaliou a leitura do texto como razoavelmente fácil ${ }^{(9)}$. Sendo assim, as pessoas sem ou com escolaridade e/ou habilidade de leitura reduzida podem desfrutar das vantagens do material escrito, pois quanto maior o valor, maior a facilidade de leitura do texto avaliado e menor o nível de escolaridade necessário para sua compreensão(18).

A validação da cartilha foi realizada a partir de um questionário online do E-survey. Após a seleção de 67 juízes, que atendessem a, pelo menos, dois dos critérios de inclusão. Foi enviado e-mail contendo a carta convite, o Termo de Consentimento Livre e Esclarecido (TCLE) e o link E-survey para acesso ao questionário. Após três rodadas de envio do questionário, 11 juízes o responderam completamente. Todos esses possuem formação em enfermagem e pósgraduação stricto sensu, sendo que 8 são enfermeiros estomaterapeutas e membros da Associação Brasileira de Estomaterapia (SOBEST) e, desses, 3 possuem experiência na construção e validação de material educativo e instrumento. 
$\mathrm{Na}$ Tabela 1, apresenta-se a síntese da análise qualitativa das recomendações realizadas pelos juízes. Todas as considerações apresentadas pelos juízes foram acatadas para a produção da versão final da cartilha educativa.

Tabela 1 - Síntese da análise qualitativa das recomendações realizadas pelos juízes. Divinópolis 2019.

\section{Sugestão dos juízes}

Página 1 Acrescentar a palavra "sobre" antes de " alguns cuidados". Retirar a palavra entre outros. Trocar "tratado" por "tratada".

Página 2 Trocar "discutir" por "conversar". Colocar dois pontos no final da fala.

Página 3 Colocar "ou antes, se sentir desconfortável" para mudança de decúbito. Colocar um relógio para antecipar a ideia a ser falada na página seguinte. Trocar a palavra "reloginho" por " relógio". Desenhar a ilustração do senhor com as pernas flexionadas e colocar o travesseiro de uma forma que os calcâneos fiquem flutuantes.

Página 4 Colocar ilustração dos ovos sobre uma superfície, frigideira por exemplo. Desenhar uma coxa de frango para demonstrar outra fonte de proteína, frutas e um copo de água. Trocar " por orientação médica ou nutricional". Aumentar o balão do pensamento e colocar dentro as imagens referidas nas páginas anteriores, em miniatura.

Página 5 Trocar "monitorize" por "observe"; "região" por "área"; " serviço de saúde" por " profissional de saúde". Desenhar enfermeira na porta da unidade de saúde para remeter vínculo.

Fonte: Elaborada pelos autores.

A cartilha foi validada quanto ao conteúdo e aparência com IVC de 0,93\% (Tabela 2). Esse valor é superior ao de $80 \%$, estipulado pelos pesquisadores para validação da cartilha, garantindo, assim, a cientificidade do conteúdo desse material e a qualidade das imagens e dos textos para interpretação pelo público alvo. Esse indicador permitiu inferir que os juízes estão em concordância sobre os aspectos do instrumento e de seus itens. Permitiu, ainda, analisar cada item, individualmente e depois o instrumento como um todo ${ }^{(19)}$.

Tabela 2 - Distribuição da validade de conteúdo (IVC), por páginas e total da cartilha educativa de autocuidado, para a prevenção de Lesão por Pressão. Divinópolis, 2019.

\begin{tabular}{lc}
\hline & IVC (\%) \\
Página 1 & 0,88 \\
Página 2 & 0,88 \\
Página 3 & 0,97 \\
Página 4 & 1 \\
Página 5 & 0,92 \\
Total & $\mathbf{0 , 9 3}$ \\
\hline
\end{tabular}

Fonte: Elaborada pelos autores.

$\mathrm{Na}$ construção de materiais educativos, reforça-se a necessidade de que os mesmos sejam interativos, atraentes e possuam linguagem adequada ao público alvo, proporcionando a troca de informações relevantes e contextualizadas ${ }^{(20)}$.

Ao desenvolver e utilizar o instrumento educativo, o profissional enfermeiro difunde seu saber técnico-científico e práticas de trabalho de forma horizontal com o paciente, que compartilha suas experiências e o saber popular. Além disso, o enfermeiro desempenha papel fundamental ao estimular e auxiliar, paciente e cuidador a se tornarem protagonistas no processo de autocuidado ${ }^{(7,21)}$.

Especificamente, no que tange à prevenção de LP, sabe-se da importância da implementação de estratégias preventivas, uma vez que o custo é menor e o risco para o paciente é praticamente inexistente ${ }^{(22)}$. Nesse sentido, reforça-se a necessidade da construção de instrumentos educativos validados que tratem de medidas 
preventivas para LP com foco nos pacientes, familiares/cuidadores.

Espera-se que os instrumentos educativos, como folhetos, manuais, folders, álbuns seriados e cartilhas possam despertar o interesse do indivíduo, fortalecer o que foi orientado, verbalmente, além de permitirem o acesso fácil para sanar dúvidas e auxiliar as ações cotidianas, contribuindo, assim, para redução na incidência e prevalência de $\mathrm{LP}^{(6)}$.

Como fator limitador do estudo, considerase o número de experts que responderam completamente o questionário.

\section{CONCLUSÃO}

A partir desse estudo, foi possível construir e validar, quanto ao conteúdo e aparência, uma cartilha de autocuidado de prevenção de LP, por meio de uma metodologia capaz de subsidiar a elaboração de uma tecnologia educativa atrativa e compreensiva. Este trabalho pode orientar a elaboração de outras cartilhas na mesma temática ou em outras da área da saúde. Espera-se que o material possa contribuir com as orientações fornecidas pelo profissional de enfermagem ao reforçar o que foi dito ao paciente e permitir que - mesmo tenha um material de fácil entendimento e acesso para sanar suas dúvidas.

O estudo oferece um material educativo de linguagem fácil e imagens que permitem o entendimento de indivíduos com menor escolaridade, com o intuito de reduzir a incidência e prevalência de $L P$, principalmente, em pacientes com maiores riscos para desenvolvê-las como idosos, cadeirantes e acamados. A produção da cartilha não exclui a necessidade de pesquisas sobre a redução da LP com acompanhamento, em longo prazo, dos pacientes e familiares/cuidadores, além de práticas inovadoras de prevenção.

\section{REFERÊNCIAS}

1- Marinho F, Passos VMDA, França EB. New century, new challenges: Changes in the burden of disease profile in Brazil. Epidemiol Serv Saúde 2016 [citado em 15 Maio 2019]; 25(4):713-24. Available http://vizhub.healthdata.org/irank/arrow.php

2- National Pressure Ulcer Advisory Panel. Pressure ulcer stages revised. Washington: NPUAP; 2016.

3- Vasconcelos JMB, Caliri MHL. Ações de enfermagem antes e após um protocolo de prevenção de lesões por pressão em terapia intensiva. Esc Anna Nery 2017;21(1):1-9. DOI: 10.5935/1414-8145.20170001

4- Smith IL, Nixon J, Brown S, Wilson L, Coleman S. Pressure ulcer and wounds reporting in NHS hospitals in England part 1: Audit of monitoring systems. J Tissue 2016;25(1):3-15. DOI: 10.1016/j.jtv.2015.11.001

5- Duarte SCM, Stipp MAC, Silva MM, Oliveira FT. Eventos adversos e segurança na assistência de enfermagem. Rev Bras Enferm. 2015;68(1):14454. DOI: 10.1590/0034-7167.2015680120p

6- Boyko TV, Longaker MT, Yang GP. Review of the current management of pressure ulcers. Adv

7- Wound Care 2018;7(2):57-67. DOI: 10.1089/wound.2016.0697

8- Soares CF, Heidemann BSI. Promoção da saúde e prevenção da lesão por pressão: Expectativas do enfermeiro da atenção primária. Texto Contexto Enferm. 2018;27(2):1-9. DOI: 10.1590/0104070720180001630016

9- Benevides JL, Coutinho JFV, Pascoal LC, Joventino ES, Martins MC, Gubert FA, et al. Construção e validação de tecnologia educativa sobre cuidados com úlcera venosa. Rev Esc Enferm USP 2016;50(2):309-16. DOI: 10.1590/S0080623420160000200018

10- Sousa LB, Hübner LC. Desafios na avaliação da compreensão leitora: Demanda cognitiva e leiturabilidade textual. Rev Neuropsicol Latinoam. 2015;7(1):34-46. DOI: 10.5579/rnl.2013.0237

11- Goldim JR. Consentimento e informação: A importância da qualidade do texto utilizado. Ver HCPA 2006 [citedado em 15 maio 2019]; 26(3):117-22. Available in: https://www.univates.br/media/Etica/leituras/Co nsentimento-e-informacao.pdf

12- Flesch R. A new readability yardstick. J Appl Psychol. 194832(3):221-33. DOI: 10.1037/h0057532

13- Costa FJ, Orsini ACR, Carneiro JS. Variações de mensuração por tipos de escalas de verificação: Uma análise do construto de satisfação discente. Rev Gestão Org. 2018;16(2):132-44. DOI: 10.21714/1679-18272018v16n2.p132-144

14- Pasquali L. Teoria e métodos de medida em ciências do comportamento. Brasília: Ministério da Educação; 1996.

15- Coluci MZO, Alexandre NMC, Milani D. Construção de instrumentos de medida na área da saúde. Ciênc Saúde Coletiva 2015;20(3):925-36. DOI: 10.1590/1413-81232015203.04332013

16- Nora CRD, Zoboli E, Vieira MM. Validação por peritos: Importância na tradução e adaptação de 
instrumentos. Rev Gaúcha Enferm. 2017;38(3):19. DOI: 10.1590/1983- 1447.2017.03.64851

17- Polit DF, Beck CT, Hungler BP, Thorell A. Fundamentos de pesquisa em enfermagem: Métodos, avaliaçao e utilizã ção. 5 ed. Porto Alegre: Artmed; 2004.

18- Medeiros RKS, Ferreira Júnior MA, Pinto $D$, Vitor AF, Santos VEP, Barichello E. Pasquali's model of content validation in the Nursing researches. Rev Enf Ref. 2015;4(4):127-35. DOI: 10.12707/RIV14009

19- Aiello CP, Lima II, Ferrari DV. Validade e confiabilidade do questionário de handicap auditivo para adultos. Braz J Otorhinolaryngol. 2011;77(4):432-40. DOI: 10.1590/S180886942011000400005

20- Alexandre NMC, Coluci MZO. Validade de conteúdo nos processos de construção e adaptação de instrumentos de medidas. Ciênc Saúde Coletiva 2011;16(7):3061-8. DOI: 10.1590/S1413-81232011000800006

21- Leite SS, Áfio ACE, Carvalho LV, Silva JM, Almeida PC, Pagliuca LMF. Construção e validação de Instrumento de Validação de Conteúdo Educativo em Saúde. Rev Bras Enferm. 2018;71(4):1732-8. DOI: 10.1590/0034-71672017-0648

22- Barbosa EMG, Sousa AAS, Vasconcelos MGF, Carvalho REFL, Oriá MOB, Rodrigues DP. Tecnologias educativas para promoção do (auto) cuidado de mulheres no pós-parto. Rev Bras Enferm. 2016;69(3):582-90. DOI: 10.1590/0034$7167.2016690323 \mathrm{i}$

23- Shi C, Dumville JC, Cullum N. Support surfaces for pressure ulcer prevention: A network metaanalysis. PLoS One 2018;13(2): e0192707. DOI: 10.1371/journal.pone.0192707

Nota: Extraído do Trabalho de Conclusão de Curso de Graduação.

Recebido em: $28 / 01 / 20$

Aprovado em: $15 / 04 / 20$

Endereço de correspondência: Daniel Nogueira Cortez Universidade Federal de São João Del-Rei, Campus Divinópolis (CCO).

Av Sebastião Gonçalves Coelho, 400

Chanadour- Sala 302.1- Bloco D

35501296 - Divinópolis, MG - Brasil

E-mail: danielcortez@ufsj.edu.br 